\title{
Performance Evaluation of IEEE 802.11 DCF Networks
}

\author{
Krzysztof Szczypiorski ${ }^{1}$, Józef Lubacz ${ }^{1}$ \\ ${ }^{1}$ Warsaw University of Technology, Institute of Telecommunications, \\ ul. Nowowiejska 15/19, 00-665 Warsaw, Poland \\ $\{k s z, j 1\} @$ tele.pw.edu.pl
}

\begin{abstract}
The paper presents a new analytical saturation throughput model of IEEE 802.11 DCF (Distributed Coordination Function) with basic access in adhoc mode. The model takes into account freezing of the backoff timer when a station senses a busy channel. It is shown that taking into account this feature of DCF is important in modeling saturation throughput - yields more accurate results than models known from literature. The proposed analytical model also takes into account the effect of transmission errors.
\end{abstract}

Keywords: WLAN, IEEE 802.11, DCF, CSMA/CA, modeling

\section{Introduction}

The paper concerns IEEE 802.11 DCF (Distributed Coordination Function), also referred to as CSMA/CA (Carrier Sense Multiple Access with Collision Avoidance), with basic access in ad-hoc mode [7]. For IEEE 802.11 DCF networks a new analytical model for throughput evaluation is proposed, assuming saturated conditions, i.e. when all stations involved in transmission have no empty queues. Saturation throughput is an efficiency measure of maximum load in saturated conditions. According to the DCF protocol, when a station senses a busy channel the backoff is suspended in effect of freezing of the station backoff timer. It is shown that taking into account this feature of DCF is important in modeling saturation throughput - yields more accurate results than models known from literature. Moreover, the influence of transmission errors is taken into account. The proposed model is based on a Markov chain.

According the authors' knowledge the first analytical model of DCF was proposed by G. Bianchi [2]. Bianchi proposed a Markov chain based model to evaluate saturation throughput, assuming a finite number of stations and ideal channel conditions (no errors).

$\mathrm{H}$. Wu et al. [18] modified Bianchi's model through introducing a limit on the number of retransmissions (maximum number of backoff stages) and a maximum size of the contention window. E. Ziouva and T. Antonakopoulos [19], and probably independently M. Ergen and P. Varaiya [5], extended Bianchi's model through taking into account freezing of the backoff timer during a busy channel occurrences. In [19] it is assumed that, after successful transmission, a station can access the medium 
without backoff; this assumption does not comply with the IEEE 802.11 standard [7]. In [5] the presented analytical solution of the introduced Markov chain is erroneous.

The above mentioned models assume ideal channel conditions, i.e. no transmission errors. P. Chatzimisios et al. [4] and Q. Ni et al. [14] extended Wu's model [18] to take account of transmission failure. In [14] ACK frames loss due to errors is taken into account; in [4] ACK frames loss is not considered.

In [2], [5] and [18] RTS/CTS (Request to Send/Clear to Send) is considered, but without taking into account the two independent retransmission counters: SLRC Station Long Transmission Retry and SSRC - Station Short Transmission Retry. In effect these models cannot be extended to take account of transmission errors. In [4] and [14] transmission errors are considered, however only for the case of basic access (i.e. only with the account of the SLRC counter).

It should be noted that in [2], [4] and [18] the authors have mistakenly taken DIFS (DCF InterFrame Space) for EIFS (Extended InterFrame Space). This mistake does not however have a very important impact on the evaluation of saturation throughput.

All the aforementioned analyses are based on Markov chains. Also other approaches were presented, e.g. in [1], [3] and [15]. These approaches make several simplifying assumptions and thus do not take into account important features of DCF.

The model presented in this paper is, generally speaking, in line with the extensions of the basic Bianchi's model [2] which were proposed in [18] and [14]. The essential difference of the presented model with respect to the latter two is in that it takes into account the effect of freezing of the stations' backoff timer along with the limitation of the number of retransmissions, maximum size of the contention window and the impact of transmission errors.

\section{The Model}

\subsection{Assumptions}

1. Saturated conditions are considered; stations have no empty queues - there is always a frame to be sent.

2. $n$ stations compete for medium access (for $n=1$ only one station sends frames to other station which can only reply with ACK).

3. Errors in the transmission medium are randomly distributed; this is the worst case for the frame error rate - FER. All stations have the same bit error rate (BER).

4. All stations are in transmission range and there are no hidden terminals.

5. Stations communicate in ad hoc mode (BSS - Basic Service Set) with basic access method.

6. All stations use the same physical layer (PHY).

7. The transmission data rate $R$ is the same and constant for all stations.

8. All frames are of constant length $L$.

9. Only data frames and ACK frames are exchanged.

10. Collided frames are discarded - the capture effect [12] is not considered. 
2.2 Saturation throughput $S$ expressed through characteristics of the physical channel

The saturation throughput $S$ is defined as in [2]:

$$
S=\frac{E[D A T A]}{E[T]}
$$

where $\mathrm{E}[D A T A]$ is the mean value of the successfully transmitted payload, and $\mathrm{E}[T]$ is the mean value of the duration of the following channel states:

$T_{I}$ - idle slot,

$T_{S}-$ successful transmission,

$T_{C}-$ transmission with collision,

$T_{E_{-} D A T A}-$ unsuccessful transmission with data frame error,

$T_{E \_A C K}-$ unsuccessful transmission with ACK error.

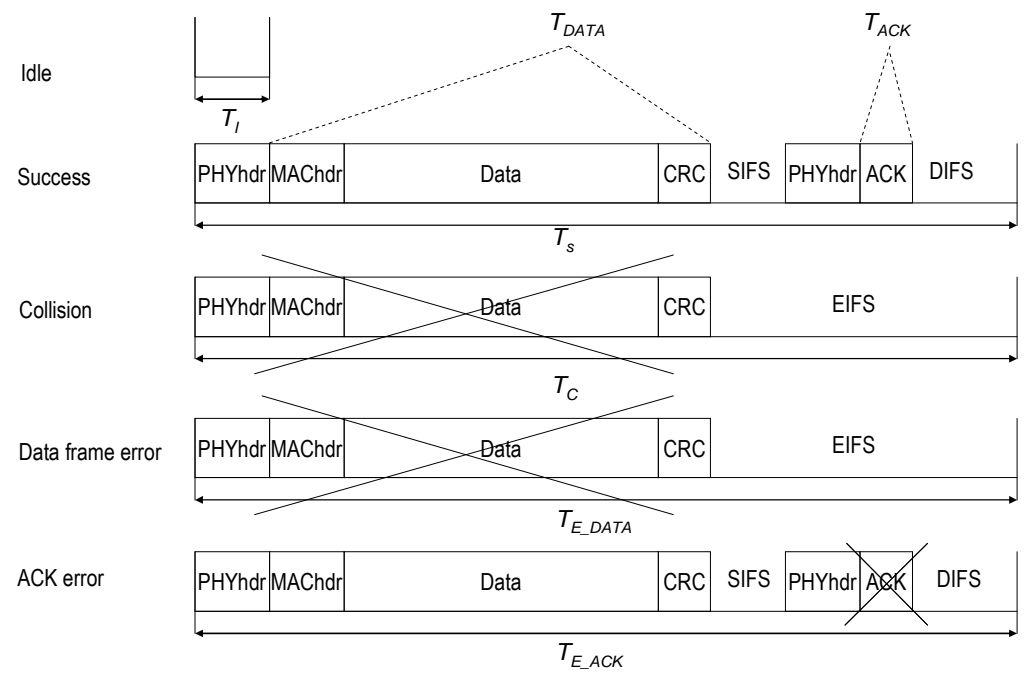

Fig. 1. States of the channel.

Fig. 1 illustrates the dependence of the above channel states on:

$T_{P H Y h d}$ - duration of a PLCP (PHY Layer Convergence Procedure) preamble and a PLCP header,

$T_{D A T A}-$ duration to transmit a data frame,

$T_{A C K}$ - duration to transmit an ACK frame,

$T_{\text {SIFS }}$ - duration of SIFS (Short InterFrame Space),

$T_{\text {DIFS }}$ - duration of DIFS,

$T_{\text {EIFS }}$ - duration of EIFS. 
The relation of the saturation throughput to physical channel characteristics is calculated similarly as in [14]:

$$
\left\{\begin{array}{l}
T_{I}=\sigma \\
T_{S}=2 T_{P H Y h d r}+T_{D A T A}+2 \delta+T_{S I F S}+T_{A C K}+T_{D I F S} \\
T_{C}=T_{P H Y h d r}+T_{D A T A}+\delta+T_{E I F S} \\
T_{E_{-} D A T A}=T_{P H Y h d r}+\delta+T_{D A T A}+T_{E I F S} \\
T_{E_{-} A C K}=T_{S}
\end{array}\right.
$$

where $\sigma$ is the duration of idle slot (aSlotTime [7]) and $\delta$ is the propagation delay.

For OFDM (Orthogonal Frequency Division Multiplexing) PHY, i.e. 802.11a [8] and 802.11g [11]:

$$
\begin{aligned}
& T_{A C K}=T_{\text {symbol }}\left\lceil\frac{L_{S E R}+L_{T A I L}+L_{A C K}}{N_{B p S}}\right\rceil \\
& T_{D A T A}=T_{\text {symbol }}\left\lceil\frac{L_{S E R}+L_{T A L L}+L_{D A T A}}{N_{B p S}}\right\rceil
\end{aligned}
$$

where:

$T_{\text {symbol }}$ - duration of a transmission symbol,

$L_{S E R}-$ ODFM PHY layer SERVICE field size,

$L_{\text {TAIL }}$ - OFDM PHY layer TAIL fields size,

$N_{B p S}$ - number of encoded bits per one symbol,

$L_{A C K}$ - size of an ACK frame,

$L_{D A T A}$ - size of a data frame.

For DSSS (Direct Sequence Spread Spectrum) PHY (i.e. 802.11 1 and 2 Mbps [7], 802.11 b [9] with long preamble) formulas (3) and (4) may be applied with $L_{S E R}=L_{T A I L}=0$ (there are no such fields). Values of $\sigma, T_{P H Y h d r}, T_{S I F S}, T_{D I F S}, T_{E I F S}, T_{\text {symbol}}$, $N_{B P S}, L_{S E R}$ and $L_{T A I L}$ are defined in accordance with 802.11 standard ([7], [8], [9], or [11]).

Probabilities corresponding to states of the channel are denoted as follows:

$P_{I}$ - probability of idle slot,

$P_{S}$ - probability of successful transmission,

$P_{C}$ - probability of collision,

$P_{E_{-} D A T A}$ - probability of unsuccessful transmission due to data frame error,

$P_{E_{-} A C K}-$ probability of unsuccessful transmission due to ACK error.

Let $\tau$ be the probability of frame transmission, $p_{e \_d a t a}$ the probability of data frame error and $p_{e \_A C K}$ the probability of ACK error. These are related to channel state probabilities as follows:

$$
\left\{\begin{array}{l}
P_{I}=(1-\tau)^{n} \\
P_{S}=n \tau(1-\tau)^{n-1}\left(1-p_{e_{-} \text {data }}\right)\left(1-p_{e_{-} A C K}\right) \\
P_{C}=1-(1-\tau)^{n}-n \tau(1-\tau)^{n-1} \\
P_{E_{-} D A T A}=n \tau(1-\tau)^{n-1} p_{e_{-} \text {data }} \\
P_{E_{-} A C K}=n \tau(1-\tau)^{n-1}\left(1-p_{e_{-} \text {data }}\right) p_{e_{-} A C K}
\end{array}\right.
$$


The saturation throughput $S$ equals

$$
S=\frac{P_{S} L_{\text {pld }}}{T_{I} P_{I}+T_{S} P_{S}+T_{C} P_{C}+T_{E_{-} D A T A} P_{E_{-} D A T A}+T_{E_{-} A C K} P_{E_{-} A C K}}
$$

where $L_{\text {pld }}$ is MAC (Medium Access Control) payload size and $L_{\text {pld }}=L-L_{\text {MAChdr }}$, where $L_{\text {MAChdr }}$ is the size of the MAC header plus the size of FCS (Frame Checksum Sequence).

$S$ can be normalized to data rate $R$ :

$$
\bar{S}=\frac{S}{R}
$$

where

$$
R=\frac{\mathrm{N}_{\mathrm{BpS}}}{T_{\text {symbol }}}
$$

As a result, saturation throughput $S$ is expressed as a function of $\tau$, $p_{e \_ \text {data }}$ and $p_{e_{\_} A C K}$. In the following sections these probabilities are evaluated.

\subsection{Probability of frame transmission $\tau$}

Let $s(t)$ be a random variable describing DCF backoff stage at time $t$, with values from set $\{0,1,2, \ldots, m\}$. Let $b(t)$ be a random variable describing the value of the backoff timer at time $t$, with values from set $\left\{0,1,2, \ldots, W_{i}-1\right\}$. These random variables are dependent because the maximum value of the backoff timer depends on backoff stage:

$$
W_{i}= \begin{cases}2^{i} W_{0}, & i \leq m^{\prime} \\ 2^{m^{\prime}} W_{0}=W_{m}, & i>m^{\prime}\end{cases}
$$

where $W_{0}$ is an initial size of contention window and $m^{\prime}$ is a maximum number by which the contention window can be doubled; $m$ ' can be both greater and smaller than $m$ and also equal to $m$. $W_{0}$ and $W_{m}$, depend on $C W_{\min }$ and $C W_{\max }$ [7]:

$$
\begin{gathered}
W_{0}=C W_{\min }+1 \\
W_{m^{\prime}}=C W_{\max }+1=2^{m^{\prime}} W_{0}
\end{gathered}
$$

The two-dimensional process $(s(t), b(t))$ will be analyzed with an embedded Markov chain (in steady state) at time instants at which the channel state changes. Let $(i, k)$ denote the state of this process. The one-step conditional state transition probabilities will be denoted by $P=(\cdot, \cdot \cdot \cdot, \cdot)$.

Let $p_{f}$ be the probability of transmission failure and $p_{\text {coll }}$ the probability of collision. The non-null transition probabilities are determined as follows (comp. Fig. 2): 

(a) $P(i, k \mid i, k+1)=1-p_{\text {coll }}, \quad 0 \leq i \leq m, 0 \leq k \leq W_{i}-2$
(b) $P(i, k \mid i, k)=p_{\text {coll }}, \quad 0 \leq i \leq m, 1 \leq k \leq W_{i}-1$
(c) $P(0, k \mid i, 0)=\left(1-p_{f}\right) / W_{0}, \quad 0 \leq i \leq m-1,0 \leq k \leq W_{0}-1$
(d) $P(i, k \mid i-1,0)=p_{f} / W_{i}, \quad 1 \leq i \leq m, 0 \leq k \leq W_{i}-1$
(e) $P(0, k \mid m, 0)=1 / W_{0}, \quad 0 \leq k \leq W_{0}-1$

Ad (a): The station's backoff timer is decremented from $k+1$ to $k$ at fixed $i$ backoff stage, i.e. the station has detected an idle slot, so the channel is idle. The probability of this event $\operatorname{Pr}\{$ channel is idle $\}=1-\operatorname{Pr}\{$ one or more station is transmitting\}. We consider saturated conditions, so Pr\{one or more station is transmitting\} equals $p_{\text {coll }}$.

Ad (b): The station's backoff timer is frozen at fixed $i$ backoff stage, i.e. the channel is busy. $\operatorname{Pr}\{$ channel is busy $\}=\operatorname{Pr}\{$ one or more station is transmitting $\}=p_{\text {coll }}$.

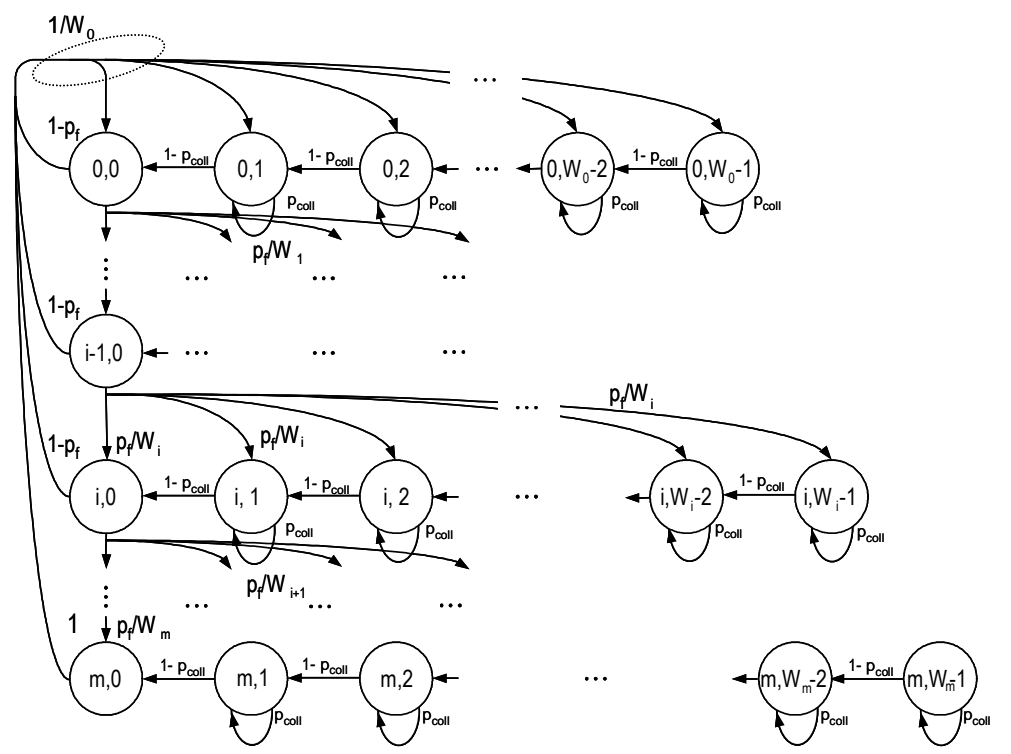

Fig. 2. Markov chain transitions.

Ad (c): The station's backoff timer is changed from 0 to $k$ and the backoff stage is changed from $i$ to 0 . The probability of this event equals: Pr\{transmission is successful and number $k$ was randomly chosen to initiate the backoff timer at stage 0\} $=\operatorname{Pr}\{$ transmission is successful\} $\}$ Pr\{number $k$ was randomly chosen to initiate the backoff timer at stage 0$\}$. The probability of successful transmission is equal to $1-p_{f}$ and the probability that number $k$ was randomly chosen to initiate the backoff timer at stage 0 equals $1 / W_{0}$.

Ad (d): The station's backoff timer is changed from 0 to $k$ and the backoff stage is changed from $i-1$ to $i$. The probability of this event equals: Pritransmission is unsuccessful and number $k$ was randomly chosen to initiate the backoff timer at stage 
$i\}=\operatorname{Pr}\{$ transmission is unsuccessful $\} \cdot \operatorname{Pr}\{$ number $k$ was randomly chosen to initiate the backoff timer at stage $i\}$. The probability of unsuccessful transmission equals $p_{f}$ and the probability that number $k$ was randomly chosen to initiate the backoff timer at stage $i$ equals $1 / W_{i}$.

Ad (e): The station's backoff timer is changed from 0 to $k$ and the backoff stage is changed from $m$ to 0 , i.e. the station has reached maximum retransmission count. The probability of this event equals the probability that number $k$ was randomly chosen to initiate the backoff timer at stage 0 , i.e. $1 / W_{0}$.

Let $b_{i, k}$ be the probability of state $(i, k)$. It can be shown that:

$$
\begin{gathered}
b_{i, 0}=p_{f} \cdot b_{i-1,0} \\
b_{i, 0}=p_{f}{ }^{i} \cdot b_{0,0}
\end{gathered}
$$

and

$$
b_{i, k}= \begin{cases}\frac{W_{i}-k}{W_{i}\left(1-p_{\text {coll }}\right)} p_{f}{ }^{i} \cdot b_{0,0}, & 0<k \leq W_{i}-1 \\ p_{f}{ }^{i} \cdot b_{0,0}, & k=0\end{cases}
$$

From:

$$
\sum_{i=0}^{m} \sum_{k=0}^{W_{i}-1} b_{i, k}=1
$$

and

$$
\sum_{i=0}^{m} b_{i, 0}=b_{0,0} \frac{1-p_{f}{ }^{m+1}}{1-p_{f}}
$$

we get

$$
b_{0,0}{ }^{-1}= \begin{cases}\frac{\left(1-p_{f}\right) W_{0}\left(1-\left(2 p_{f}\right)^{m+1}\right)-\left(1-2 p_{f}\right)\left(1-p_{f}{ }^{m+1}\right)}{2\left(1-2 p_{f}\right)\left(1-p_{f}\right)\left(1-p_{\text {coll }}\right)}+\frac{1-p_{f}{ }^{m+1}}{1-p_{f}} & m \leq m^{\prime} \\ \frac{\Psi}{2\left(1-2 p_{f}\right)\left(1-p_{f}\right)\left(1-p_{\text {coll }}\right)}+\frac{1-p_{f}{ }^{m+1}}{1-p_{f}}, & m>m^{\prime}\end{cases}
$$

where

$$
\Psi=\left(1-p_{f}\right) W_{0}\left(1-\left(2 p_{f}\right)^{m^{\prime}+1}\right)-\left(1-2 p_{f}\right)\left(1-p_{f}^{m+1}\right)+W_{0} 2^{m^{\prime}} p_{f}^{m^{\prime}+1}\left(1-2 p_{f}\right)\left(1-p_{f}^{m-m^{\prime}}\right)
$$

The probability of frame transmission $\tau$ is equal to $\operatorname{Pr}\{$ backoff timer equals 0$\}$ and thus:

$$
\begin{gathered}
\tau=\sum_{i=0}^{m} b_{i, 0}= \\
= \begin{cases}\left(\frac{\left(1-p_{f}\right) W_{0}\left(1-\left(2 p_{f}\right)^{m+1}\right)-\left(1-2 p_{f}\right)\left(1-p_{f}{ }^{m+1}\right)}{2\left(1-2 p_{f}\right)\left(1-p_{f}\right)\left(1-p_{\text {coll }}\right)}+\frac{1-p_{f}{ }^{m+1}}{1-p_{f}}\right)^{-1} \frac{1-p_{f}{ }^{m+1}}{1-p_{f}}, & m \leq m^{\prime} \\
\left(\frac{\Psi}{2\left(1-2 p_{f}\right)\left(1-p_{f}\right)\left(1-p_{\text {coll }}\right)}+\frac{1-p_{f}{ }^{m+1}}{1-p_{f}}\right)^{-1} \frac{1-p_{f}{ }^{m+1}}{1-p_{f}}, & m>m^{\prime}\end{cases}
\end{gathered}
$$

For $p_{\text {coll }}=0$ the above solution is the same as presented in [14]. 


\subsection{Probability of transmission failure $p_{f}$ and probability of collision $p_{\text {coll }}$}

The probability of transmission failure

$$
p_{f}=1-\left(1-p_{\text {coll }}\right)\left(1-p_{e}\right)
$$

where $p_{e}$ is the frame error probability:

$$
p_{e}=1-\left(1-p_{e_{-} d a t a}\right)\left(1-p_{e_{-} A C K}\right)
$$

where $p_{e_{-} \text {data }}$ is FER for data frames and $p_{e_{-} A C K}$ is FER for ACK frames. $p_{e_{-} \text {data }}$ and $p_{e \_A C K}$ can be calculated from bit error probability (i.e. BER) $p_{b}$ :

$$
\begin{aligned}
& p_{e_{-} \text {data }}=1-\left(1-p_{b}\right)^{L_{\text {data }}} \\
& p_{e_{-} A C K}=1-\left(1-p_{b}\right)^{L_{A C K}}
\end{aligned}
$$

The probability of collision

$$
p_{\text {coll }}=1-(1-\tau)^{n-1}
$$

Finally

$$
p_{f}=1-\left(1-p_{\text {coll }}\right)\left(1-p_{e}\right)=1-(1-\tau)^{n-1}\left(1-p_{e}\right)
$$

Equations (20) and (26) form a non-linear system with two unknown variables $\tau$ and $p_{f}$ which may be solved numerically.

\section{Validation}

The presented model was validated with the use of simulation in two steps. The aim of Step 1 was to compare the proposed model with (i) models presented in [2] and [18] in which channel errors are not taken into account, with (ii) the special case of model [14] for which BER is assumed zero, and with (iii) simulations (also presented in [6]). In Step 2, channel errors are taken into account; the accuracy of the presented model is evaluated with simulations presented in [13] and compared to the model presented in [14].

\section{Step 1}

The $n s-2$ simulator version 2.29 [17] was used. The IEEE 802.11 DSSS $1 \mathrm{Mbps}$ PHY was simulated (OFDM PHY is not implemented in the standard version of $n s-2$ ). The simulation was performed for saturated conditions with static routing and for 1000 bytes MAC frames UDP traffic. The results are presented in Table 1 and Fig. 3.

The proposed model was also compared with simulation results presented in [6], which were obtained with the simulation tool created at Universitat Politècnica de Catalunya in Barcelona [15]. In Table 2 the condition of simulation and simulation results are presented. Note that non-aggregated values of saturation throughput $(S / n)$ are presented. 
Table 1. Normalized values of saturation throughput for IEEE 802.11 DSSS 1 Mbps with $L=1000$ bytes and $B E R=0$.

\begin{tabular}{|c|c|c|c|c|c|c|}
\hline $\boldsymbol{n}$ & $\begin{array}{c}\text { Bianchi } \\
\text { model } \\
{[\mathbf{2}]}\end{array}$ & $\begin{array}{c}\text { Wu } \text { et al. } \\
\text { model } \\
{[\mathbf{1 8}]}\end{array}$ & $\begin{array}{c}\text { Ni } \text { et al. } \\
\text { model } \\
{[\mathbf{1 4}]}\end{array}$ & $\begin{array}{c}\text { Proposed } \\
\text { model }\end{array}$ & $\begin{array}{c}\text { Simulation } \\
\text { (average) }\end{array}$ & $\begin{array}{c}\text { Standard } \\
\text { deviation }\end{array}$ \\
\hline 1 & 0.8769 & 0.8769 & 0.8769 & 0.8769 & 0.8780 & 0.000 \\
\hline 2 & 0.8666 & 0.8666 & 0.8657 & 0.8661 & 0.8635 & 0.000 \\
\hline 4 & 0.8329 & 0.8329 & 0.8306 & 0.8367 & 0.8354 & 0.003 \\
\hline 10 & 0.7602 & 0.7586 & 0.7540 & 0.7779 & 0.7625 & 0.005 \\
\hline 20 & 0.6929 & 0.6846 & 0.6783 & 0.7238 & 0.7200 & 0.002 \\
\hline 30 & 0.6497 & 0.6330 & 0.6258 & 0.6891 & 0.6872 & 0.004 \\
\hline 50 & 0.5904 & 0.5558 & 0.5477 & 0.6421 & 0.6303 & 0.003 \\
\hline 80 & 0.5297 & 0.4684 & 0.4599 & 0.5955 & 0.5633 & 0.004 \\
\hline
\end{tabular}

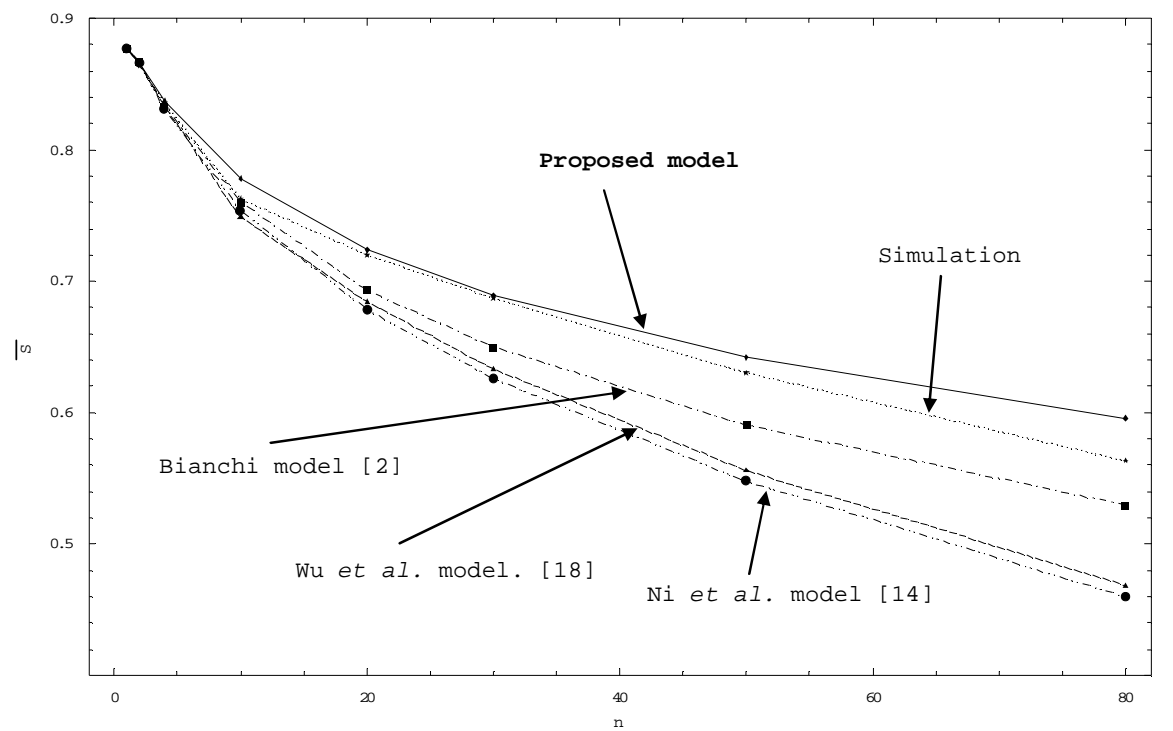

Fig. 3. Normalized saturation throughput: analytical and simulation results for IEEE 802.11 DSSS 1 Mbps with $L=1000$ bytes and $B E R=0$.

Table 2. Non-aggregated values (in Mbps) of saturation throughput for IEEE 802.11g $54 \mathrm{Mbps}$ ERP-OFDM with $L=1500$ bytes and $B E R=0$.

\begin{tabular}{|c|c|c|c|c|c|}
\hline $\boldsymbol{n}$ & $\begin{array}{c}\text { Bianchi } \\
\text { model } \\
{[\mathbf{2}]}\end{array}$ & $\begin{array}{c}\text { Wu } \text { et al. } \\
\text { model } \\
{[\mathbf{1 8}]}\end{array}$ & $\begin{array}{c}\text { Ni } \text { et al. } \\
\text { model } \\
{[\mathbf{1 4}]}\end{array}$ & $\begin{array}{c}\text { Proposed } \\
\text { model }\end{array}$ & Simulation \\
\hline 1 & 31.36 & 31.36 & 31.36 & 31.36 & 31.79 \\
\hline 2 & 16.24 & 16.24 & 16.15 & 16.05 & 16.18 \\
\hline 4 & 7.90 & 7.90 & 7.79 & 7.86 & 7.85 \\
\hline 10 & 2.87 & 2.86 & 2.79 & 2.93 & 2.92 \\
\hline 15 & 1.82 & 1.78 & 1.72 & 1.88 & 1.87 \\
\hline 20 & 1.30 & 1.26 & 1.21 & 1.36 & 1.36 \\
\hline 25 & 1.00 & 0.95 & 0.91 & 1.06 & 1.06 \\
\hline 50 & 0.43 & 0.37 & 0,35 & 0.47 & 0.49 \\
\hline 100 & 0.17 & 0.11 & 0.10 & 0.21 & 0.22 \\
\hline
\end{tabular}


Step 2

Although the $n s-2$ simulator enables to simulate channel errors, the mechanism of errors occurrence is based on physical features and is thus different from the one assumed in the proposed analytical model (randomly distributed bit errors). For this reason the $n s-2$ simulator was not used.

The accuracy of the proposed model was compared with results obtained by solving the model presented in [14] for assumptions concerning the physical layer and its parameters which are presented in Table 3 and Fig. 4. The table also presents simulation results which were obtained by the authors of [13] (according to [15], the authors used the same simulation tool as authors of [6], i.e. the simulator mentioned above). In these simulations a random pattern of bit-error occurrence was assumed (i.e. as assumed in the model presented in this paper).

Table 3. Normalized values of saturation throughput for IEEE 802.11g $54 \mathrm{Mbps}$ with $L=1500$ bytes and $B E R=10^{-5}$ and $B E R=10^{-4}$.

\begin{tabular}{|c|c|c|c|c|c|c|}
\hline \multirow[t]{2}{*}{$n$} & $\begin{array}{c}\text { Ni et al. } \\
\text { model [14] }\end{array}$ & $\begin{array}{c}\text { Proposed } \\
\text { model }\end{array}$ & Simulation & $\begin{array}{c}\text { Ni et al. } \\
\text { model [14] }\end{array}$ & $\begin{array}{c}\text { Proposed } \\
\text { model }\end{array}$ & Simulation \\
\hline & \multicolumn{3}{|c|}{ BER $=10^{-5}$} & \multicolumn{3}{|c|}{ BER $=10^{-4}$} \\
\hline 2 & 0.5246 & 0.5207 & 0.5080 & 0.1425 & 0.1412 & 0.0711 \\
\hline 4 & 0.5148 & 0.5167 & 0.4989 & 0.1640 & 0.1619 & 0.0993 \\
\hline 10 & 0.4672 & 0.4880 & 0.4739 & 0.1699 & 0.1705 & 0.1365 \\
\hline 15 & 0.4354 & 0.4693 & 0.4593 & 0.1640 & 0.1682 & 0.1461 \\
\hline 20 & 0.4081 & 0.4541 & 0.4476 & 0.1564 & 0.1648 & 0.1517 \\
\hline 25 & 0.3843 & 0.4413 & 0.4383 & 0.1486 & 0.1612 & 0.1537 \\
\hline 50 & 0.2906 & 0.3965 & 0.4052 & 0.1128 & 0.1459 & 0.1572 \\
\hline 100 & 0.1656 & 0.3448 & 0.3607 & 0.0619 & 0.1260 & 0.1504 \\
\hline
\end{tabular}

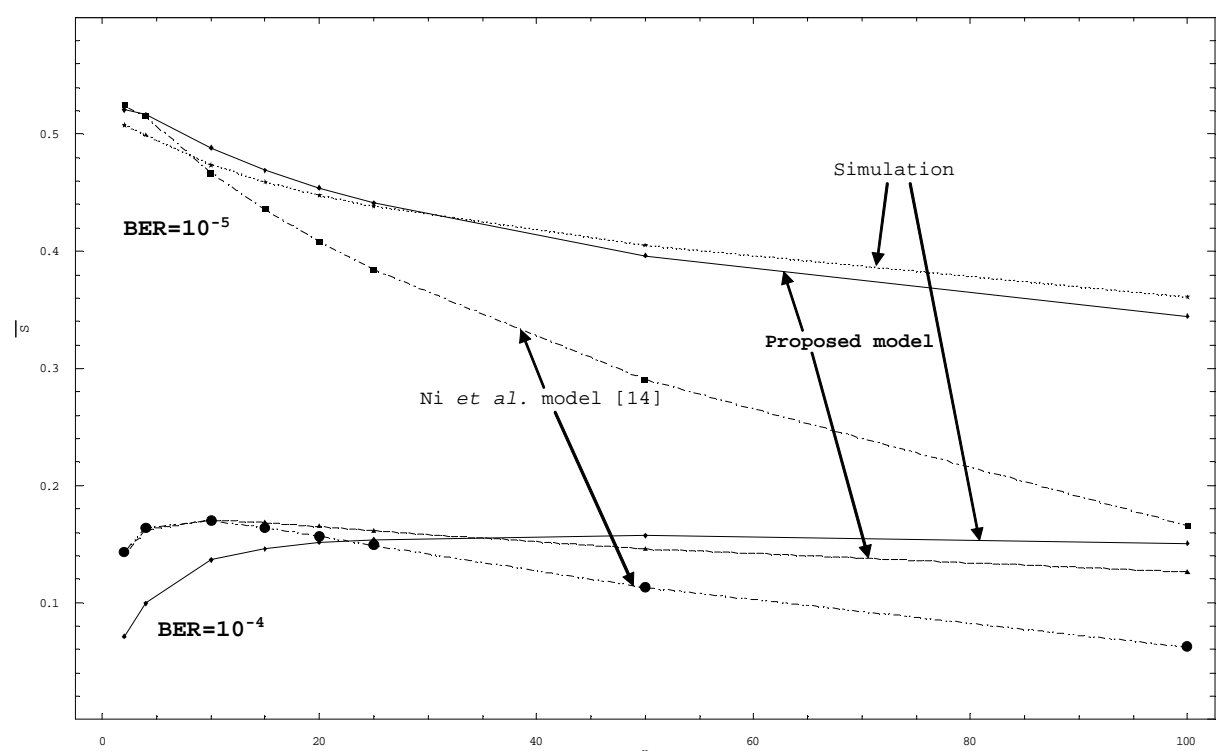

Fig. 4. Normalized saturation throughput: analytical and simulation results for IEEE $802.11 \mathrm{~g}$ 54 Mbps ERP-OFDM with $L=1500$ bytes and $B E R=10^{-5}$ and $B E R=10^{-4}$. 
To conclude: the results presented in the tables and figures above show that the proposed model has good accuracy both in the case of error-free and error-prone channels. For error-free conditions the model yields some overestimation while other models known from literature tend to underestimate the saturation throughput. For both error-free and error-prone cases the proposed model shows better accuracy than the literature models with which it was compared, especially for large number of stations. The latter is the consequence of the fact that the proposed model takes into account freezing of the backoff timer; the impact of the freezing of the backoff timer on throughput evaluation increases with the increase of the number of station competing for access to the transmission medium.

Future work could be focused on taking into account such features of the IEEE 802.11 protocol as the RTS/CTS and EDCA (Enhanced Distributed Channel Access) [10].

\section{References}

1. Bianchi, G., Tinnirello, I.: Remarks on IEEE 802.11 DCF Performance Analysis. IEEE Communications Letters, Vol. 9 (2005) 765-767

2. Bianchi, G.: Performance Analysis of the IEEE 802.11 Distributed Coordination Function. IEEE Journal on Selected Areas in Communications, Vol. 18, No. 3 (2000) 535-547

3. Cali, F., Conti, M., Gregori, E.: Dynamic Tuning of the IEEE 802.11 Protocol to Achieve a Theoretical Throughput Limit. IEEE/ACM Trans. Networking, Vol. 8, No. 6 (2000) 785-799

4. Chatzimisios, P., Boucouvalas, A., Vitsas, V.: Influence of Channel BER on IEEE 802.11 DCF. IEE Electronics Letters, Vol. 39, No. 23 (2003)

5. Ergen, M., Varaiya, P.: Throughput Analysis and Admission Control in IEEE 802.11a. Springer Mobile Networks and Applications, Vol. 10, No. 5 (2005) 705-706

6. Heusse, M., Rousseau, F., Guillier, R., Duda, A.: Idle Sense: An Optimal Access Method for High Throughput and Fairness in Rate Diverse Wireless LANs. In: SIGCOMM'05 Conference on Applications, Technologies, Architectures and Protocols for Computer Communications, Philadelphia (2005) 121-132

7. IEEE 802.11, 1999 Edition (ISO/IEC 8802-11: 1999) IEEE Standards for Information Technology - Telecommunications and Information Exchange between Systems - Local and Metropolitan Area Network - Specific Requirements - Part 11: Wireless LAN Medium Access Control (MAC) and Physical Layer (PHY) Specifications (1999)

8. IEEE 802.11a-1999 (8802-11:1999/Amd 1:2000(E)), IEEE Standard for Information technology - Telecommunications and information exchange between systems - Local and metropolitan area networks - Specific requirements - Part 11: Wireless LAN Medium Access Control (MAC) and Physical Layer (PHY) specifications - Amendment 1: Highspeed Physical Layer in the $5 \mathrm{GHz}$ band (1999)

9. IEEE 802.11b-1999 Supplement to 802.11-1999, Wireless LAN MAC and PHY specifications: Higher speed Physical Layer (PHY) extension in the $2.4 \mathrm{GHz}$ band (1999)

10. IEEE 802.11e-2005, IEEE Standard for Information technology - Telecommunications and information exchange between systems - Local and metropolitan area networks Specific requirements Part 11: Wireless LAN Medium Access Control (MAC) and Physical Layer (PHY) specifications: Amendment 8: Medium Access Control (MAC) Quality of Service Enhancements (2005)

11. IEEE 802.11g-2003 IEEE Standard for Information technology - Telecommunications and information exchange between systems - Local and metropolitan area networks - 
Specific requirements - Part 11: Wireless LAN Medium Access Control (MAC) and Physical Layer (PHY) specifications - Amendment 4: Further Higher-Speed Physical Layer Extension in the $2.4 \mathrm{GHz}$ Band (2003)

12. Kochut, A., Vasan, A., Shankar, A., Agrawala, A.: Sniffing Out the Correct Physical Layer Capture Model in 802.11b. In: 12th IEEE International Conference on Network Protocols (ICNP 2004), Berlin (2004)

13. Lopez-Aguilera, E., Heusse, M., Rousseau, F., Duda, A., Casademont, J.: Evaluating Wireless LAN Access Methods in Presence of Transmission Errors. In: IEEE INFOCOM. Barcelona (2006)

14. Ni, Q., Li, T., Turletti, T., Xiao, Y.: Saturation Throughput Analysis of Error-Prone 802.11 Wireless Networks. Wiley Journal of Wireless Communications and Mobile Computing (JWCMC), Vol. 5, Issue 8 (2005) 945-956

15. Private communication with Prof. Andrzej Duda

16. Tay, Y., Chua, K.: A Capacity Analysis for the IEEE 802.11 MAC Protocol. Wireless Networks, Vol. 7, No. 2 (2001) 159-171

17. The Network Simulator - ns-2. URL: http://nsnam.isi.edu/nsnam/index.php/Main_Page

18. Wu, H., Peng, Y., Long, K., Cheng, S., Ma, J.: Performance of Reliable Transport Protocol over IEEE 802.11 Wireless LAN: Analysis and Enhancement. In: IEEE INFOCOM 02 (2002)

19. Ziouva, E., Antonakopoulos, T.: CSMA/CA Performance under High Traffic Conditions: Throughput and Delay Analysis. Computer Communications, Vol. 25 (2002) 313-321 